\title{
Determining the rate of microbially- mediated pyrrhotite dissolution using integrated geochemical, magnetic, and genomic analyses
}

K. K. HobArT ${ }^{1,2 *}$, J. M. FEINBERG ${ }^{1,2}$, D. S. JONES ${ }^{3}$, J.V. BAILEY ${ }^{1}$

${ }^{1}$ Department of Earth \& Environmental Sciences, University of Minnesota, Minneapolis, Minnesota 55455

(*correspondence: hobar010@umn.edu)

(feinberg@umn.edu) (baileyj@umn.edu)

${ }^{2}$ Institute for Rock Magnetism, University of Minnesota, Minneapolis, Minnesota 55455

${ }^{3}$ Department of Earth and Environmental Science, New Mexico Institute of Mining and Technology, Socorro, NM 87801 (daniel.s.jones@nmt.edu)

Pyrrhotite $\left(\mathrm{Fe}_{1-\mathrm{x}} \mathrm{S}, 0 \leq \mathrm{x} \leq 0.125\right)$ is the second most abundant iron sulfide mineral in the Earth's crust, and is frequently associated with copper, nickel, and platinum-group element ores. Understanding pyrrhotite dissolution under environmental conditions is critical to improving management of mine waste and water. However, little is known about the influence of microorganisms on the rate of pyrrhotite dissolution, particularly at near-neutral $\mathrm{pH}$.

We have conducted laboratory experiments using isolated sulfur-oxidizing microorganisms and enrichment cultures obtained from sulfide-bearing rocks from Minnesota's Duluth Complex, which hosts a large, undeveloped disseminated $\mathrm{Cu}-$ Ni-PGE deposit. The presence of these organisms results in a substantial acceleration of pyrrhotite dissolution as compared to abiotic conditions. Different isolates and enrichment communities result in different dissolution rates and affect the amount of elemental sulfur formed and the precipitation of secondary iron minerals. These differences in secondary minerals can be characterized using low- and hightemperature magnetic techniques with higher sensitivities than traditional geochemical or mineralogical techniques.

Small subunit 16S rRNA gene sequencing of the enrichment experiments, as well as whole genome sequencing of several isolates, allows us to link the differences in dissolution rates to strain or community specific elements. We found that enrichments with sulfur- and iron-oxidizing members only slightly increased rates over sulfur-oxidizing isolates, although less elemental sulfur was produced in incubations with the enrichments. Among the sulfuroxidizing isolates, the fastest rates were realized with a strain of Sulfuriferula that has a somewhat minimal sulfur-oxidation pathway consisting of only a partial SOX system and sulfide quinone oxidoreductases. 